\title{
Chlamydia trachomatis infection in infants: a prospective study
}

\author{
P M PREECE, ${ }^{*} \mathrm{~J}$ M ANDERSON,* AND R G THOMPSON $\dagger$ \\ ${ }^{*}$ New Cross Hospital and + Public Health Laboratory Service, Wolverhampton
}

SUMMARY In a prospective study of Chlamydia trachomatis infection in pregnancy, 198 mothers positive for chlamydial antigen were identified; the infants of 174 were followed for up to six months and $C$ trachomatis was recovered in cell culture from 43 infants $(25 \%)$. Conjuctivitis occurred in significantly more infants who were positive for $C$ trachomatis $(20$ of $43,47 \%)$ than in those who were negative $(18$ of $131,14 \%)$. There were also significantly more lower respiratory tract infections among infants with positive cultures (six of 43, 14\%, compared with three of 131, $2 \%$ ). The chlamydial antigen enzyme linked immunosorbent assay (ELISA) was positive in 61 of 131 infants from whom $C$ trachomatis was not recovered in cell culture. False positive results were usually associated with the isolation of Staphylococcus aureus from samples of pharyngeal aspirate.

Our results confirm that $C$ trachomatis infection is a common cause of neonatal conjunctivitis, and respiratory infection in the first few months of life, with an incidence of $8 \cdot 2 / 1000$ live births. Because the infection is easily treated by oral erythromycin, however, screening during pregnancy is not warranted.

Chlamydia trachomatis infection is commonly transmitted from mother to infant in the perinatal period and is now recognised as the commonest cause of neonatal conjunctivitis. ${ }^{1}$ It is also a significant cause of lower respiratory tract infection in infants 3-4 months old ${ }^{2}$ and may be an important pathogen in otitis media. ${ }^{4}$ Prospective studies in the United States have reported carriage rates in pregnancy of from 2 to $18 \%$. ${ }^{5-9}$ Transmission occurs in $28-61 \%$ of infants of mothers positive for $C$ trachomatis. The incidence of $C$ trachomatis infection in infants has not been documented in Britain, so the value of antenatal screening and treatment in reducing infant morbidity is not known.

We have previously reported the findings of a prospective study of $C$ trachomatis infection in pregnancy in an obstetric population in a district general hospital and explored the maternal risk factors associated with infection and their association with perinatal morbidity and mortality. ${ }^{10}$ In the present study we outline the natural history of $C$ trachomatis infection in infants, the rate of transmission, complications of infection, and the persistence of carriage. Such data are necessary to assess the morbidity associated with $C$ trachomatis in the infant population so that the benefits of an antenatal screening programme may be predicted.

\section{Patients and methods}

In a prospective study of $C$ trachomatis infection in pregnancy, 3309 unselected women attending a district general hospital obstetric unit, between 1 September 1985 and 31 August 1986, were screened for $C$ trachomatis infection on presentation in labour. ${ }^{10}$ An endocervical swab was taken during routine vaginal and speculum examination using a cotton tipped plastic swab. Swabs were placed in transport media, stored at $4^{\circ} \mathrm{C}$, and transported to the laboratory. Chlamydial antigen was detected using the Boots Celltech monoclonal antibody enzyme linked immunosorbant assay (ELISA).

As a result of this study 198 women positive for chlamydial antigen were identified. They were offered treatment for themselves and their partners, and invited to bring their infants to the paediatric clinic for follow up. Infants were seen at 3,6, 12, and 26 weeks. They were examined for evidence of conjunctivitis, respiratory infection, and any other abnormality. At each visit pharyngeal aspirate and cotton tipped plastic swabs from the lower conjunctivae were collected and transported immediately to the laboratory, in chlamydia transport media. ${ }^{11}$

The samples were examined for the presence of chlamydial antigen by a monoclonal antibody 
ELISA. Samples were also inoculated into cell culture medium containing Buffalo green monkey cells as previously described. ${ }^{11}$ The diagnosis was based on the identification of characteristic intracellular inclusion bodies on Giemsa stained monolayers of cells. If all samples were negative on the ELISA on at least two consecutive occasions no further samples were collected and it was assumed that the organism had not been transmitted, though the infants were followed up to act as controls. If the presence of chlamydial antigen was confirmed by the detection of inclusion bodies in cell culture transmission of $C$ trachomatis had occurred.

Infants with respiratory symptoms were investigated to exclude other causal organisms by routine microbiological and virus culture, and immunofluorescence for respiratory syncitial virus, of the nasopharyngeal aspirate. Infants with conjunctivitis were also investigated for other bacterial pathogens.

Infants treated with erythromycin or tetracycline by their general practitioners before investigation were excluded from the study. Symptomatic infants who were positive for $C$ trachomatis were treated with tetracycline oily eye drops for conjunctivitis. Erythromycin $(30 \mathrm{mg} / \mathrm{kg} /$ day $)$ was added for those who did not respond to tetracycline alone, and was also used to treat infants with lower respiratory tract infections.

\section{Results}

During a one year period 198 mothers positive for chlamydial antigen were identified at risk of transmitting $C$ trachomatis to their infants. Seven infants who had been treated with erythromycin or tetracycline before their first follow up visit were excluded, and 16 did not attend for follow up; the parents of one child refused to take part in the study. Infants of 174 mothers positive for chlamydial antigen were therefore followed up. Six further infants were excluded during the study because they were treated with either erythromycin or tetracycline in circumstances outside the protocol.

$C$ trachomatis was cultured on at least one occasion from at least one site in $43(25 \%)$ of the 174 infants (table 1), and was isolated from two infants who were delivered by elective caesarean section despite intact membranes. It was isolated from the nasopharyngeal aspirate alone in 21 infants, from conjunctival swabs alone in eight, and from both sites in 14.

$C$ trachomatis was isolated from 32 of 174 infants $(18 \%)$ at 3 weeks, decreasing to two of 92 infants $(2 \%)$ at 26 weeks (figure). Infants who received antibiotics have been excluded from this figure. This suggests gradual loss of carriage in infants who acquired the organism but received no treatment. Of the 43 infants who acquired $C$ trachomatis at any time in the study eight were positive for the first

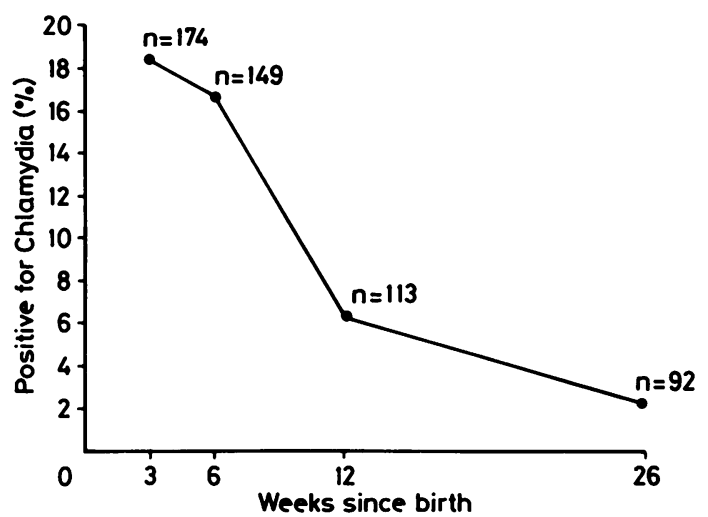

Figure Prevalence of Chlamydia trachomatis infection in 174 infants exposed to maternal infection.

Table 1 Complications in 174 infants exposed to maternal Chlamydia trachomatis infection

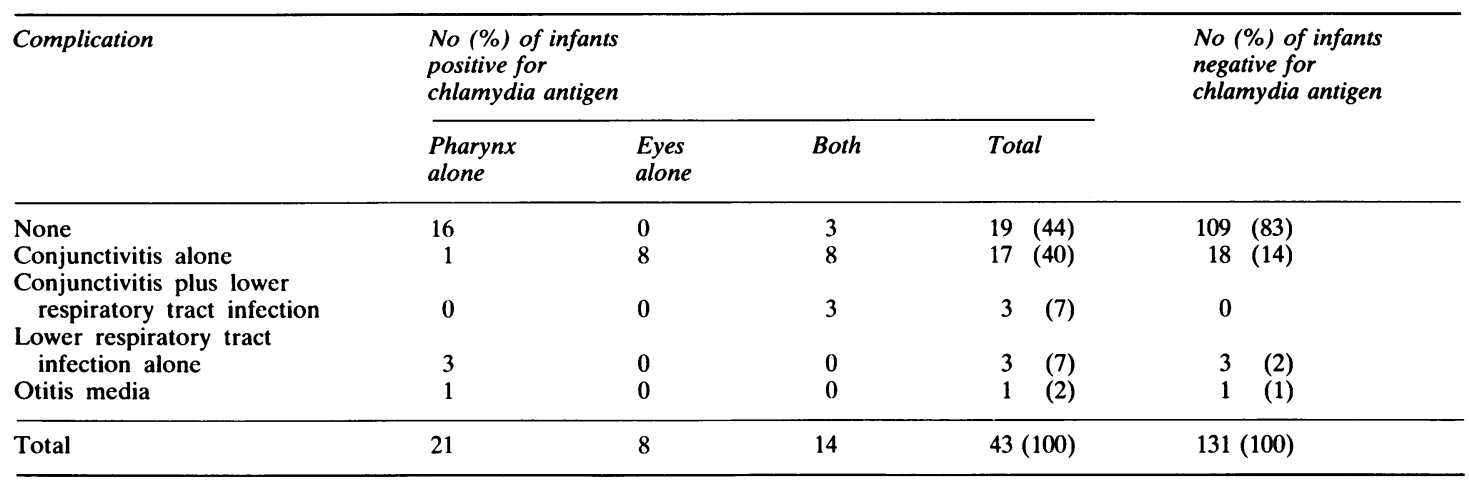


time at six weeks, two at 12 weeks, and one at 26 weeks. In the last three cases chlamydial antigen was detected at previous visits and only small numbers of organisms were recovered from cell culture.

\section{NEONATAL CONJUNCTIVITIS}

Conjunctivitis occurred in $20(46.5 \%)$ of the 43 infants positive for $C$ trachomatis, although the organism could not be cultured from conjunctival swabs in one (table 1). In eight cases the conjunctivitis was mild and resolved before the results of conjunctival swabs were available; it therefore required no treatment. In 12 cases it was sufficiently severe to require specific treatment; in five tetracycline eye drops alone, one erythromycin alone, and in six oral erythromycin was added because of failure of the tetracycline to clear the infection. The conjunctivitis resolved in all cases with or without treatment, and no long term effects were apparent at six months. Only one case presented before discharge from the maternity unit at one week, the remainder presenting at one to three weeks; the mean age at presentation was 14 days.

Bacterial conjunctivitis occurred in only $18(14 \%)$ of 131 infants negative for $C$ trachomatis, which is significantly less than among the infants who were positive $(p<0 \cdot 0005)$. These cases were clinically less severe and responded promptly to local treatment with antibiotics. There were no cases of gonococcal conjunctivitis in either group.

\section{RESPIRATORY TRACT INFECTIONS}

Six infants positive for $C$ trachomatis $(14 \%)$ developed lower respiratory tract infections characterised by tachypnoea, hyperinflation, indrawing, and wheeze between 4 and 6 weeks. In each case $C$ trachomatis was grown from phanygeal aspirates at the time of the illness. Three had previously had conjunctivitis; one had been treated with local tetracycline and in the other two the conjunctivitis had resolved without treatment. In one, respiratory syncytial virus shown by immunofluorescence in addition to the $C$ trachomatis. All suffered mild respiratory symptoms, but only one infant required admission to hospital and radiographic changes were minor, consisting only of hyperinflation.

Three infants negative for $C$ trachomatis $(2 \%)$ had lower respiratory tract infections during the study $(p<0.005)$; no organisms were isolated in this group. $C$ trachomatis associated respiratory tract infections resolved promptly in all infants, in two spontaneously and in four after oral treatment with erythromycin. Two infants (one from each group) suffered recurrent purulent otitis media with perforation. In one infant Haemophilus influenzae was isolated from the ear swabs and $C$ trachomatis from pharyngeal aspirate but not from ear swabs. Treatment with amoxycillin was unsuccessful but she responded to erythromycin.

\section{USE OF THE ELISA IN CHILDREN}

The ELISA test was positive in 61 of the 131 infants in whom $C$ trachomatis was not subsequently isolated in cell culture; in 53 of these $S$ aureus was also isolated. In one infant a false positive ELISA was associated with the isolation of Candida albicans. False positive results were therefore common, particularly in pharyngeal aspirates. The results of this test alone were not sufficient to support the diagnosis of $C$ trachomatis infection.

\section{Discussion}

The present prospective study has shown that infection with $C$ trachomatis is transmitted to the neonate in a quarter of pregnancies complicated by this organism. In two cases this occurred despite intact membranes, confirming that this is not an unusual occurrence. ${ }^{12}$ This rate of transmission gives rise to an estimate of neonatal infection of $14 \cdot 7 / 1000$ in our population (table 2). Symptomatic infection occurred in 24 of $43(56 \%)$ infants, which represents an incidence of $8 \cdot 2 / 1000$. These figures are underestimates, as some of the 24 infants excluded (seven because of undocumented treatment by general practitioners) probably had symptomatic $C$ trachomatis infection.

C trachomatis was recovered by cell culture in most infants in the first six weeks of life. The number of positive cultures declined with time, suggesting a gradual loss of chlamydial carriage as a commensal organism, in a similar manner to other perinatally acquired organisms such as Mycoplasma hominis or Ureaplasma urealyticum. ${ }^{13}$ Interestingly,

Table 2 Prevalence of maternal and neonatal Chlamydia trachomatis infection

\begin{tabular}{lrr}
\hline & & $\begin{array}{c}\text { Prevalencel } \\
\text { 1000 live births }\end{array}$ \\
\hline No of mothers screened & 3309 & \\
No of mothers with chlamydia infection & 198 & 60 \\
No of infants followed up & 174 & \\
No of infants positive for chlamydia & 43 & $14 \cdot 7$ \\
No of infants with conjunctivitis & 20 & $6 \cdot 8$ \\
No of infants with lower respiratory & & \\
tract infections & 6 & $2 \cdot 1$ \\
No of infants with otitis media & 1 & $0 \cdot 3$ \\
No of infants with sympatomatic & & \\
chlamydial infection & 24 & $8 \cdot 2$ \\
No of infants with asymptomatic & 19 & $6 \cdot 5$ \\
chlamydial infection & 19 & \\
\hline
\end{tabular}


in three infants the cell culture was not positive until at least 12 weeks. Presumably only small numbers of organism were acquired at birth and time was required for the organism to replicate sufficiently to yield a positive result in cell culture. Postnatal acquisition, however, cannot be excluded.

$C$ trachomatis is now the commonest cause of neonatal conjunctivitis. ${ }^{114}$ The incidence in this study is higher than that previously reported, ${ }^{15} 16$ but this could be due either to a different study design or to a real increase in the prevalence of maternal carriage of the organism.

Presentation before the second week of life is rare, so most children are initially treated by their general practitioners. If the condition is recognised promptly and treated appropriately, response is complete and late complications are rare. Oral erythromycin is now the treatment of choice for $C$ trachomatis conjunctivitis because it is excreted in tears. ${ }^{17}$ It also eradicates nasopharyngeal carriage so preventing the respiratory complications that occurred in three of our cases. Ocular prophylaxis with local erythromycin has been attempted in some units in the United States but it is not completely effective in preventing conjunctivitis and in no way alters the incidence of $C$ trachomatis infection in the respiratory tract. ${ }^{18}$

Respiratory infections in the first four months are frequently caused by $C$ trachomatis. ${ }^{3}$ The rate in this study is an underestimate as seven children with conjunctivitis were treated with systemic antibiotics and so were no longer susceptible to $C$ trachomatis respiratory infection, so the true rate was six of 36 $(17 \%)$. As these seven children suffered the most severe conjunctivitis, the rate in untreated infants may even be higher. The clinical pattern may be indistinguishable from infection with respiratory syncytial virus except that there is no seasonal variation in incidence. Mixed infections may also occur so other respiratory pathogens (including other perinatally acquired organisms such as cytomegalovirus) should be sought. ${ }^{313}$

We found the ELISA a highly efficient method of screening women for chlamydial infection. It was also specific in infants with conjunctivitis, and false positive reactions were rare. ${ }^{1}$ We do not at present recommend the test for the routine investigation of children with respiratory symptoms, as false positive reactions occurred frequently in pharyngeal aspirates (usually due to cross reactivity with $S$ aureus). ${ }^{19}$ Cell culture remains the most reliable method of diagnosis for samples of respiratory secretions though the specificity of the ELISA may be improved in the future (RG Thompson, personal communication). Direct immunofluorescence using monoclonal antibodies may also be used for the diagnosis of $C$ trachomatis infection. ${ }^{1}$ We did not use this method as we wished to assess a method that avoided both cell culture and microscopy compared with the standard method.

Screening in pregnancy to identify mothers at risk of transmitting $C$ trachomatis to their offspring has been successfully carried out, and treatment in late pregnancy significantly reduces the risk of neonatal transmission. ${ }^{20-22}$ We have doubts, however, about the cost effectiveness of screening as maternal chlamydial infection has no effect on perinatal mortality, ${ }^{10}$ only $14 \%$ of infants of infected mothers suffered symptomatic infestions, gradual loss of asymptomatic carriage occurs spontaneously, and treatment of symptomatic infants is safe and effective. Paediatricians and general practitioners must, however, have a high index of suspicion of chlamydial infection in infants as it is now the commonest cause of neonatal conjunctivitis and an important cause of respiratory infection during the first four months of life.

We thank our research assistant, June Sperring, the nurses of the childrens' day unit, and the staff of the microbiology department. Advice on computer analysis was given by Robert Lancashire of the department of social medicine, University of Birmingham, and the study was funded by the Wolverhampton Perinatal Research Fund and Abbott Laboratories.

\section{References}

1 Rapoza PA, Quinn TC, Kiessling R, Green R, Taylor H. Assessment of neonatal conjunctivitis with a direct immunofluorescent monoclonal antibody stain for chlamydia. JAMA 1986;255:3369-73.

2 Beam MO, Saxon EM. Respiratory tract colonisation and a distinctive syndrome in infants with $\mathrm{C}$ trachomatis. $N$ Engl J Med 1977;296:306-10.

${ }^{3}$ Paisley JW, Laver BA, McIntosh K, Glode MP, Schachter J, Rumach C. Pathogens associated with acute lower respiratory tract infection in young children. Pediatr Infect Dis 1984;3: $15-9$.

4 Banks JR, Vanderdriesen G, Stark E. Chlamydia trachomatis in smears from eyes, ears and throats of children with chronic otitis media. Lancet 1985;ii:278.

5 Frommell GT, Rothenberg R, Warg S, McIntosh K. Chlamydial infection of mothers and their infants. $J$ Pediatr 1979;95:28-32.

6 Hammerschlag MR, Anderka M, Semine DZ, McComb D, McCormack W. Prospective study of maternal and infantile infection with Chlamydia trachomatis. Pediatrics 1979;64:142-8.

7 Heggie AD, Lumicas GG, Stuart LA, Gyveo MT. Chlamydia trachomatis infections in mothers and infants. Am J Dis Child 1981;135:507.

${ }^{8}$ Grossman M, Schachter J, Sweet R, Bishop E, Jordan C. Prospective studies in Chlamydia in newborns. In: Mardh PA, Holmes KK, Oriel JD, eds. Chlamydial infections. Amsterdam: Elsevier, 1982:213-6.

${ }^{9}$ Schachter J, Grossman M, Sweet RL, Holt J, Jordan C, Bishop E. Prospective study of perinatal transmission of $\mathrm{C}$. trachomatis. JAMA 1986;255:3374-7.

10 Preece PM, Ades A, Thompson RG, Brooks JH. Chlamydia trachomatis infection in late pregnancy: a prospective study. Paediatric and Perinatal Epidemiology 1989 (in press).

11 Willis PJ, Johnson L, Thompson RG. Isolation of Chlamydia 
using McCoy and Buffalo green monkey cells. J Clin Pathol 1984;37:120-1.

12 Givner LB, Rennels MB, Woodward C, Huang S. Chlamydia trachomatis infection in an infant delivered by caesarean section. Pediatrics 1981;68:420-1.

13 Stagno S, Brasfield DM, Brown MB, et al. Infant pneumonitis associated with cytomegalovirus, chlamydia, pneumocystis and ureaplasma: a prospective study. Pediatrics 1981;68:322-9.

14 Winceslaus J, Goh BT, Dunlop EMC, et al. Diagnosis of ophthalmia neonatorum. Br Med J 1987;295:1377-9.

15 Prentice MJ, Hutchinson GR, Taylor-Robinson D. A microbiological study of neonatal conjunctive and conjunctivitis. $\mathrm{Br} J$ Ophthalmol 1977;61:601-7.

${ }^{16}$ Pierce JM, Ward ME, Seal DV. Ophthalmia neonatorum in the 1980s: incidence, aetiology and treatment. $\mathrm{Br} J$ Ophthalmol 1982;66:728-31.

17 Rettig PJ. Chlamydial infections in pediatrics: diagnostic and therapeutic considerations. Pediatr Infect Dis 1986;5:158-62.

${ }^{18}$ Hammerschlag MR, Chandler JW, Alexander ER, et al. Erythromycin ointment for occular prophylaxis of neonatal chlamydial infection. JAMA 1980;244:2291-3.
${ }^{19}$ Krech T, Gerhard-Fsadni D, Hoffman N, Miller SM. Interference of Staphylococcus aureus in the detection of Chlamydia trachomatis by monoclonal antibodies. Lancet 1985;i:1161-2.

20 Podgare JK, Betts R, Alden E, Alexander ER. Effectiveness of maternal third trimester erythromycin in prevention of infant $C$. trachomatis infection. Proceedings of the 20th Interscience Conference in Antimicrobial Agents and Chemotherapy. New Orleans: American Society of Microbiology 1980:524.

${ }^{21}$ Schachter J, Sweet RL, Grossman M, Landers D, Robbie M, Bishop E. Experience with routine use of erythromycin for Chlamydia infection in pregnancy. $N$ Engl $J$ Med 1986;314: 276-9.

22 McMillan JA, Wiener LB, Lamberson HV, et al. Efficacy of maternal screening and therapy in the prevention of Chlamydial infection of the newborn. Infection 1985;13:263-6.

Correspondence to Dr PM Preece, The Children's Hospital, Ladywood Middleway, Birmingham B16 8ET.

Accepted 18 November 1988 